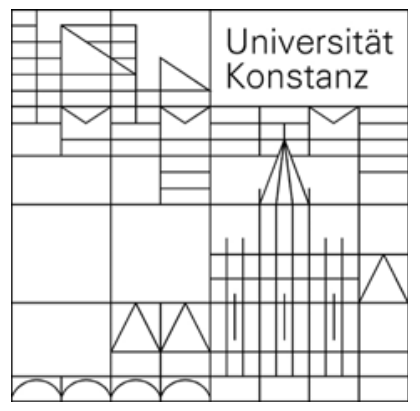

Rechts-, Wirtschafts- und

Verwaltungswissenschaftliche

Sektion

Fachbereich

Wirtschaftswissenschaften

Mathias Kifmann

Risk Selection and

Complementary Health

Insurance:

The Swiss Approach

Diskussionsbeiträge 


\title{
Risk Selection and Complementary Health Insurance: The Swiss Approach
}

\author{
Mathias Kifmann ${ }^{* \dagger}$
}

January 2005

\begin{abstract}
A common approach to avoid risk selection in health insurance markets is to define and enforce a community-rated basic benefit package. The market for complementary insurance is usually completely separate from the market for basic health insurance. In Switzerland, however, the basic benefit package and complementary insurance are offered by the same insurer. Risk-based premiums are allowed with respect to complementary insurance. In this paper, the Swiss integration approach is compared to the separation approach. It is shown that under the integration approach insurers creamskim by selling complementary insurance to low risks at a discount. Nevertheless, the integration approach can be Pareto-superior if the cost savings due to the integration of basic and complementary insurance are sufficiently large.
\end{abstract}

JEL-classification: H51, I18.

Keywords: health insurance, risk selection, complementary insurance

\footnotetext{
*Universität Konstanz, Fach D 136, 78457 Konstanz, Germany; Email: Mathias.Kifmann@uni-konstanz.de.

${ }^{\dagger}$ I thank Konstantin Beck, Friedrich Breyer and Normann Lorenz for helpful comments.
} 


\section{Introduction}

In most industrialized countries citizens obtain basic health insurance coverage through a public health insurance system where premiums are not allowed to depend on the health status. Further medical services can be obtained through complementary health insurance. Whether the basic and the complementary coverage can be purchased from the same insurer depends on the regulation of the health insurance market. In Austria and Germany, for example, basic and complementary coverage are completely separated, i.e. they must be offered by different insurers. Insurers which offer the basic coverage charge community-rated premiums whereas insurers selling complementary insurance risk-adjust their premiums. In Switzerland, however, basic and complementary insurance are allowed to be integrated. Insurers who offer the basic coverage as well as other insurers can sell complementary coverage. For the basic coverage, insurers are obliged to charge a uniform premium. Premiums for the complementary coverage, however, are risk-based.

Both, the separating solution practiced in Austria and Germany and the Swiss integration approach, have their drawbacks. On the one hand, separating basic and complementary insurance is likely to raise the administrative costs of providing health care. The insured must sign separate contracts which causes additional transaction costs. Furthermore, costs may be raised due to the necessity of processing claims separately. Finally, spill-over effects can arise if the consumption of the complementary benefits influences the consumption of the basic benefits (Danzon (2002, p. 926)). On the other hand, the integration approach suffers from a different problem if, as is the case in Switzerland, insurers are competing for the insured. Since they are restricted to charge a uniform premium for the basic coverage, they have an incentive to cream-skim, i.e. to attract low risk and to deter high risk individuals (Pauly (1984)). The pricing of the complementary benefit can be a means to do so. In particular, low-risk types are likely to receive favorable offers. ${ }^{1}$

In this paper, the benefits and drawbacks of the two approaches are compared within a theoretical model. It is assumed that the cost of the complementary benefit is higher when it is purchased from a separate insurer. This tends to make the integration approach superior. Furthermore, insurers are able to identify the risktype and can therefore use the price of the complementary benefit to attract low

\footnotetext{
${ }^{1}$ Cream-skimming can also occur if insurers must charge uniform premiums but are allowed to offer the complementary benefit besides the basic benefit (see Kifmann (2002)).
} 
risks. This possibility favors the separation approach. In an equilibrium analysis, the prices for the basic and the complementary benefit under each approach are derived and then compared.

The paper is structured as follows. Section 2 discusses the organization of basic and complementary coverage in the Swiss health insurance system. In Section 3 , the model is presented. Section 4 derives the allocation under the separating approach. In Section 5, the integration approach is analyzed. The two approaches are compared in Section 6. The effects of setting a minimum price for complementary insurance are discussed in Section 7. Finally, Section 8 summarizes the results and concludes.

\section{Basic and complementary coverage in the Swiss health insurance system}

In Switzerland, the basic benefit package covers expenditure for ambulatory care, drugs and hospital stays. Dental care is not included with a few exceptions. Furthermore, a deductible of 300 Swiss Francs and 10\% coinsurance up to a limit of 700 Swiss Francs are compulsory. In the market for basic mandatory insurance, insurers are required to charge a uniform premium and to accept any applicant. Citizens have a free choice between nonprofit sickness funds. In 1998, the market for basic health insurance accounted for two thirds of total health insurance expenditure. $^{2}$

Complementary insurance covers special hotel services in case of hospitalization and supplementary coverage for benefits not included in the mandatory package. ${ }^{3}$ These benefits include the possibility to be treated outside the canton of residence, coverage of additional drugs, alternative medicine and access to head physicians. Complementary insurance is offered by both sickness funds and private for-profit insurers. Both can adjust premiums to risk and refuse to cover high risks. In case an individual does not disclose all health and medical conditions, insurers can terminate coverage for complementary insurance. In 1998, a fifth of the popula-

\footnotetext{
${ }^{2}$ See Colombo (2001, p. 24).

${ }^{3}$ See Colombo (2001, p. 22). Mossialos et al. (2002, p. 60-61) make a distinction between (i) complementary coverage that provides full or partial cover for services not fully covered by basic insurance and (ii) supplementary insurance which gives access to superior accommodation and amenities in a hospital. Complementary health insurance in Switzerland contains elements of both definitions.
} 
tion bought complementary insurance. Expenditure for complementary coverage was $30.9 \%$ of total health insurance expenditure. Sickness funds had a market share of $74 \% .{ }^{4}$ According to the survey by the Bundesamt für Sozialversicherung (2000), $7 \%$ of individuals with complementary health insurance obtained it from an insurer different from the sickness fund providing basic coverage.

Concerns that complementary health insurance can be used for risk-selection have been expressed by Beck (1998) and Colombo (2001). In particular, health insurers providing basic coverage may give a discount to low risks. This incentive exists as long as expected costs for the basic benefit package are below the uniform premium quoted by the insurer. A consequence is that the cross-subsidies for high risks in the basic benefit package are reduced.

In addition, high risks may be disadvantaged when buying complementary coverage. If insurers providing basic coverage can offer complementary coverage at lower costs than competitors because of lower transaction costs, they may be unwilling to pass on these gains to high risk individuals since these are unattractive in the first place. Nevertheless, high risk types do not pay more for complementary insurance than under the separation approach as long as they can obtain complementary coverage from other insurers. As administrative costs are saved, however, competition between insurers may translate possible mark-ups for complementary coverage for high risks into a lower price for basic coverage.

\section{The model}

We assume that there are two health benefits, the basic benefit and the complementary benefit. Individuals are characterized by the utility they derive from each service and the expected cost of the service. It is assumed that there are two cost types $i=L, H$ with cost $c^{i}$ for the basic and costs $C^{i}$ for the complementary benefit. The proportion of $L$-types is $\lambda$ where $0<\lambda<1$. The $L$-types are less costly with respect to both benefits.

\section{Assumption 1 - Definition of risk types:}

$$
c^{L}<c^{H} \text { and } C^{L}<C^{H} .
$$

\footnotetext{
${ }^{4}$ See Colombo (2001, p. 9 and p. 24).
} 


\begin{tabular}{ccc} 
Type & $V$ & Fraction \\
\hline$L 0$ & $V^{0}=0$ & $\lambda(1-\mu)$ \\
$L 1$ & $V^{1}>0$ & $\lambda \mu$ \\
$H 0$ & $V^{0}=0$ & $(1-\lambda)(1-\mu)$ \\
$H 1$ & $V^{1}>0$ & $(1-\lambda) \mu$
\end{tabular}

Table 1: Distribution of types

For the basic benefit, we assume that everyone derives the same utility $v$ and make

\section{Assumption 2 - Preference for the basic benefit:}

$$
v>c^{H} .
$$

This assumption guarantees that it is always efficient that all individuals receive the basic benefit.

With respect to the complementary benefit, there are two preference types. A fraction $\mu$ derives positive utility $V=V^{1}>0$ from the complementary benefit. The fraction $1-\mu$ derives no utility, i.e. $V=V^{0}=0$. Thus, there are four types $i j, i=L, H, j=0,1$. Assuming that the cost types $i$ and the preference types $j$ are distributed independently, the fraction of each type is given in Table 1.

Total utility of an individual of type $i j$ is given by

$$
u^{i j}(k)=v+k V^{j}-e^{i j}(k), \quad k=0,1,
$$

where $k$ equals 0 if the individual only chooses the basic benefit and 1 if she also obtains the complementary benefit. The expenditure for health insurance is $e^{i j}(k)$ and depends on how the health insurance market is regulated. It is assumed that an individual buys the complementary benefit if $u^{i j}(1) \geq u^{i j}(0)$.

The health insurance market is perfectly competitive. Health insurers are risk neutral and maximize expected profits. ${ }^{5}$ They organize the provision of health care. ${ }^{6}$ Insurers are able to identify the cost-type and can therefore, if allowed by the regulator, charge risk-based premiums. In the following, basic insurers

\footnotetext{
${ }^{5}$ Although the Swiss health insurance market is mainly served by nonprofit insurers, this assumption is a reasonable working hypothesis. Even if insurers cannot officially make profits, their managers can be interested in generating a surplus for their salary and fringe benefits.

${ }^{6}$ In this model, there is no insurance function due to risk neutrality of the insured. See Glazer and McGuire (2000) and Kifmann (2002) for similar approaches which focus on the organizational role of health insurers.
} 
are insurers which offer the basic benefit and, if permitted, the complementary benefit. These insurers are obliged to accept any applicant and to charge a uniform premium for the basic benefit. Furthermore, everybody must purchase the basic benefit from a basic insurer.

The cost of the complementary benefit depends on whether it is offered by the basic insurer or by another insurer. If a basic insurer offers the complementary benefit, the cost is $C^{i}$. If it is offered by another insurer, then there are additional administrative costs $a$ per contract. ${ }^{7}$ These are due to the costs of setting up a separate contract and the necessity of processing claims separately. It is therefore desirable that the basic and complementary insurance are purchased from one insurer. In the following, we assume that it is always efficient that individuals with a positive preference for the complementary benefit receive it.

\section{Assumption 3 - Preference for the complementary benefit:}

$$
V^{1}>C^{H}+a \text {. }
$$

The regulator's aim is to ensure that everyone has access to the basic benefit at a price independent of the risk-type. This implies that he wants to redistribute in favor of $H$-types because these would have to pay higher risk-based premiums for the basic benefit. Since only a fraction of the population benefits from the complementary benefit, however, redistribution with respect to the complementary benefit is not regarded as desirable. Ideally, the regulator could use type-dependent transfers to redistribute between the types. ${ }^{8}$ However, we assume in the following that this is beyond his administrative capacity. Instead he tries to reach his objective by regulating the health insurance market.

\section{The separation approach}

Under the separation approach, the complementary benefit cannot be used for risk-selection. Abstracting from other possibilities of risk-selection, perfect competition between health insurers implies that the equilibrium price for the basic benefit, $\hat{p}$, must be equal to average costs of the basic benefit:

$$
\hat{p}=\bar{c} \equiv \lambda c^{L}+(1-\lambda) c^{H} .
$$

\footnotetext{
${ }^{7}$ The administrative cost of providing the basic benefit are already included in $c^{i}$.

${ }^{8}$ See Pauly, Danzon, Feldstein, and Hoff (1992).
} 
Insurers who offer the complementary benefit charge risk-based premiums $P^{i}$. Due to the administrative cost $a$, the fair premium is

$$
P^{i}=C^{i}+a, \quad i=L, H
$$

An individual will buy complementary insurance if and only if $V^{j} \geq C^{i}+a$. Following Assumption 3, individuals with a positive preference $V^{1}$ will therefore opt for the complementary benefit.

We summarize our results in

Proposition 1: Under the separation approach, everyone obtains the basic benefit at price $\hat{p}=\bar{c}$. The price for the complementary benefit is $P^{i}=C^{i}+a, i=L, H$.

\section{The integration approach}

For the integration approach, we suppose the following sequence of events:

1. Insurers set price $p$ for the basic benefit.

2. Individuals contact the basic insurer. For the complementary benefit, basic insurers make individual offers $P^{i}$.

3. Individuals decide whether to buy only the basic benefit or the basic and the complementary benefit from an insurer.

4. All insurers offer the complementary benefit to those individuals who bought only the basic benefit from another insurer at stage 3 .

This sequence of events is motivated by the fact that insurers must publicly quote a price for the basic benefit. A price offer for the complementary benefit is then made when an individual contacts the insurer.

For stage 2, we assume that the price offer $P^{i}$ cannot be lower than a minimum price $m>0$. This rules out that insurers give away the complementary insurance or even pay low risk individuals to accept complementary insurance. Therefore $C^{L}-m$ is the maximum discount which can be given to L1-types. 
At stage 4, the assumption of perfect competition in the health insurance market implies that insurers will offer the complementary benefit at price $C^{i}+a$. Thus, administrative costs $a$ correspond to the maximum mark-up that a basic insurer can possibly charge when selling the complementary benefit to $H 1$-types at stage 2 .

In the Appendix, we derive the equilibrium prices. There, we first analyze the price offer at stage 2 for a given price $p$. We assume that individuals buy the complementary benefit from their basic insurer if indifferent. Then we turn to stage 1 in which insurers set the price $p$ in anticipation of their price offers at stage 2 and derive the equilibrium price $p^{*}$, i.e. the lowest price yielding zero expected profits, and the implied prices $P^{H}$ and $P^{L}$ for the complementary benefit. We obtain

Propositon 2: Under the integration approach, the equilibrium price for the basic benefit is

$$
p^{*}=\left\{\begin{array}{lll}
\min \left\{p_{1}, p_{2}\right\} & \text { if } \quad c^{H}-c^{L}>\min \left\{\frac{a}{\lambda},\left[\frac{1-\mu}{\lambda}+\mu\right] a+\mu\left(C^{L}-m\right)\right\} \\
\min \left\{p_{3}, p_{4}\right\} & \text { if } \quad c^{H}-c^{L} \leq \min \left\{\frac{a}{\lambda},\left[\frac{1-\mu}{\lambda}+\mu\right] a+\mu\left(C^{L}-m\right)\right\}
\end{array}\right.
$$

where the prices $p_{1}$ to $p_{4}$ are given by

$$
\begin{aligned}
& p_{1}=\bar{c}+\frac{\mu(1-\lambda)}{1-\mu \lambda}\left[\lambda\left(c^{H}-c^{L}\right)-a\right] \\
& p_{2}=\bar{c}+\mu\left[\lambda\left(C^{L}-m\right)-(1-\lambda) a\right] \\
& p_{3}=\bar{c} \\
& p_{4}=\bar{c}+\frac{\mu \lambda}{1-\mu(1-\lambda)}\left[C^{L}-m-(1-\lambda)\left(c^{H}-c^{L}\right)\right] .
\end{aligned}
$$

Individuals always buy the complementary benefit from their basic insurer. The equilibrium prices for the complementary benefit are

$$
\begin{array}{ll}
P_{1}^{H}=C^{H}+a & P_{1}^{L}=c^{L}+C^{L}-p_{1} \\
P_{2}^{H}=C^{H}+a & P_{2}^{L}=m \\
P_{3}^{H}=c^{H}+C^{H}-p_{3} & P_{3}^{L}=c^{L}+C^{L}-p_{3} \\
P_{4}^{H}=c^{H}+C^{H}-p_{4} & P_{4}^{L}=m .
\end{array}
$$


Thus, the equilibrium is characterized by four different combinations of prices for the basic and complementary benefit. In the following, we refer to these as cases (1) to (4). To interpret Proposition 2 we first discuss when basic insurers

1. charge the maximum mark-up $a$ when selling the complementary benefit to $H 1$-types (cases (1) and (2)), i.e. set price $P^{H}=C^{H}+a$,

2. give the maximum discount $C^{L}-m$ for the complementary benefit to $L 1$ types (cases (2) and (4)), i.e. set price $P^{L}=m$.

\section{Maximum mark-up for H1-types}

In cases (1) and (2), insurers charge the maximum price for the complementary benefit for $H 1$-types and therefore make profit $a$ per contract. As the condition for cases (1) and (2)

$$
c^{H}-c^{L}>\min \left\{\frac{a}{\lambda},\left[\frac{1-\mu}{\lambda}+\mu\right] a+\mu\left(C^{L}-m\right)\right\}
$$

shows this case arises

- if the administrative cost $a$ is small. Reformulating condition (5) yields

$$
a<\max \left\{\lambda\left(c^{H}-c^{L}\right) ; \frac{\lambda\left(c^{H}-c^{L}\right)-\mu \lambda\left(C^{L}-m\right)}{1-\mu(1-\lambda)}\right\} \equiv \tilde{a} .
$$

For values of $a$ above $\tilde{a}$, the mark-up cannot be enforced. Insurers can enter the market and make $H 1$-types a fair offer with the total price $p_{3}+P_{3}^{h}=c^{H}+C^{H}$ as in cases (3) and (4).

- if the cost difference for the basic benefit $c^{H}-c^{L}$ or the share of low risk types $\lambda$ are large. In this case, the difference between cost $c^{H}$ and average costs $\bar{c}$ is large. Basic insurers can charge the maximum markup $a$ for the complementary benefit because any fair offer by another insurer for complete coverage at total price $c^{H}+C^{H}$ is more expensive and would not be chosen by $H 1$-types.

The profits at stage 2 by charging the mark-up are dissipated by competition at stage 1 . Therefore the equilibrium prices $p_{1}$ and $p_{2}$ are decreasing in $a$. This effect is increasing in the share of individuals with a preference for the complementary benefit $\mu$ and the share of high risks $1-\lambda$. We state this result in

Corollary 2.1: A mark-up a for high risk types reduces the equilibrium prices for the basic benefit. 


\section{Maximum discount $C^{L}-m$ for L1-types}

In cases (2) and (4), insurers charge the minimum price $m$ for the complementary benefit for $L 1$-types. Thus, they obtain the maximum discount $C^{L}-m$. Using $p^{*}=\min \left\{p_{1}, p_{2}\right\}$ from Proposition 2 yields the condition for case (2)

$$
p_{2}<p_{1} \Leftrightarrow c^{H}-c^{L}>\frac{1-\mu \lambda}{1-\lambda}\left(C^{L}-m\right)+\mu a .
$$

Likewise, from $p^{*}=\min \left\{p_{3}, p_{4}\right\}$ we can derive a condition for case (4) or

$$
p_{4}<p_{3} \quad \Leftrightarrow \quad c^{H}-c^{L}>\frac{1}{1-\lambda}\left(C^{L}-m\right) .
$$

Thus, cases (2) and (4) are more likely,

- the larger $c^{H}-c^{L}$ and the smaller the share of low risks $\lambda$. In this case, the difference between average costs $\bar{c}$ and costs $c^{L}$ is large which gives insurers a strong incentive to sell the complementary benefit at a discount.

- the smaller the maximum discount $\left(C^{L}-m\right)$ which can be given to $L 1$-types. This makes it more likely that the constraint $P^{l} \geq m$ will bind.

In addition, low administrative costs $a$ make it more likely that insurers give the maximum discount (case (2) rather than case (1)) because the crosssubsidies between on $H$ and $L$-types cannot sufficiently be reduced by the mark-up for the complementary benefit for $H 1$-types.

The discount to $L 1$-types is financed indirectly through the premiums for the basic benefit. This explains why the equilibrium prices $p_{2}$ and $p_{4}$ are increasing in $C^{L}-m$. We summarize this result in

Corollary 2.2: A discount $C^{L}-m$ to low risk types raises the equilibrium prices for the basic benefit.

So far, we have not commented on case (3). Here basic insurers are not restricted by the maximum mark-up or the maximum discount and make $L 1$ - and $H 1$-types are totally fair offer with $p_{3}+P_{3}^{i}=c^{i}+C^{i}$. Thus, cross-subsidies between $L 1$ - and $H 1$-types vanish completely. The remaining cross-subsidies between $L 0$ - and $H 0$ types lead to $p_{3}=\bar{c}$. Case (3) arises for a small cost difference $c^{H}-c^{L}$, i.e. small 
possible cross-subsidies. Furthermore, a large maximum mark-up $a$ and a large maximum discount $\left(C^{L}-m\right)$ make case (3) more likely because then insurers have ample scope to reduce cross-subsidies.

Next, we turn to the role of $\mu$, i.e. the share of individuals valuing the complementary benefit. The possible equilibrium prices in (4) show that for $\mu=0$, i.e. without individuals who value the complementary benefit, we simply obtain the result that everybody buys the basic benefit at a price equal to average costs: setting $\mu=0$ in (5) implies $p_{1}=p_{2}=p_{3}=p_{4}=\bar{c}$. If $\mu>0$, then the equilibrium

price is generally different from $\bar{c}$ unless case (3) arises. From (4), it can be seen that

$$
\frac{\partial p_{z}}{\partial \mu}\left\{\begin{array}{ll}
>0 & \text { if } p_{z}>\bar{c} \\
<0 & \text { if } p_{z}<\bar{c}
\end{array} \quad, \quad z=1,2,3,4 .\right.
$$

Thus, an increase in $\mu$ amplifies the difference to $\bar{c}$ as more $L 1$ - and H1-types can be discriminated by discounts and mark-ups.

Finally, note that the result that individuals always buy the complementary benefit from their basic insurer fits quite well with the finding by the Bundesamt für Sozialversicherung (2000) that only $7 \%$ of individuals with complementary health insurance purchased it from an insurer different from their basic insurer. The small difference can be explained by other factors from which the model abstracts, e.g. innovative complementary insurance which is not available from the basic insurer.

\section{Comparing the regimes}

Is $p^{*}$, the price for the basic benefit under the integration approach, higher or lower than $\hat{p}=\bar{c}$, the price under the separation approach? The answer depends on which case arises:

(1) In this case, we have

$$
p_{1} \gtreqless \bar{c} \quad \Leftrightarrow \quad \lambda\left(c^{H}-c^{L}\right) \gtreqless a .
$$

Case (1) is only possible if $c^{H}-c^{L}>a / \lambda$ which implies $\lambda\left(c^{H}-c^{L}\right)>a$. Therefore, we must have $p_{1}>\bar{c}$.

(2) Here we find

$$
p_{2} \gtreqless \bar{c} \quad \Leftrightarrow \quad a \lesseqgtr \frac{\lambda}{1-\lambda}\left(C^{L}-m\right) \equiv \hat{a} .
$$

No general conclusion is possible. The answer depends on the relative size of $\lambda, C^{L}-m$ and $a$. 
(3) In this case, we have $p_{3}=\bar{c}$.

(4) Here the condition is

$$
p_{4} \gtreqless \bar{c} \quad \Leftrightarrow \quad C^{L}-m \gtreqless(1-\lambda)\left(c^{H}-c^{L}\right) .
$$

Condition $p^{*}=\min \left\{p_{3}, p_{4}\right\}$ implies

$$
C^{L}-m<(1-\lambda)\left(c^{H}-c^{L}\right)
$$

Thus in case (4), we have $p_{4}<\bar{c}$.

Of particular importance are the maximum discount and the maximum mark-up: The integration approach leads to a lower price for the basic benefit

- if the maximum discount $C^{L}-m$ is small and therefore the reduction of cross-subsidies from $L 1$-types is limited (see conditions (8) and (9)).

- the maximum mark-up $a$ is large (see condition (8) and the necessary condition $a \geq \tilde{a}$ for case (4)). This mark-up corresponds to savings in administrative costs compared to the separation approach since individuals always buy complementary insurance from their basic insurer. Competition at stage 1 dissipates these profits and lowers the price for the basic benefit. In particular, we can state the following result

Proposition 3: If the maximum mark-up falls in the range $\hat{a}<a<\tilde{a}$, then the equilibrium price for the basis benefit is lower under the integration approach.

Proof: If $a<\tilde{a}$, then only cases (1) and (2) can arise. For $a>\hat{a}$, we have $p_{2}<\bar{c}$. Since $p^{*}=\min \left\{p_{1}, p_{2}\right\}$ if $a<\tilde{a}$ (see conditions (5) and (6)) and $p_{1}>\bar{c}$, we must have $p^{*}=p_{2}<\bar{c}$.

Thus, if the maximum mark-up is sufficiently high, the price for the basic benefit may fall below average costs. This is illustrated in Figure 1 which shows how the equilibrium price of the basic benefit depends on $a$ if the conditions for Proposition 3 are fulfilled. It is based on the parameters $c^{H}=200, c^{L}=100, C^{H}=50$, $C^{L}=25, \lambda=0.5, \mu=0.5$ and $m=5$. These parameters imply $\bar{c}=150, \hat{a}=20$, $\tilde{a}=60$. Cases (2) and (4) arise. Insurers give $L 1$-types the maximum discount and are restricted by $P^{L} \geq m$. Thus, $L 1$-types continue to pay cross-subsidies. 


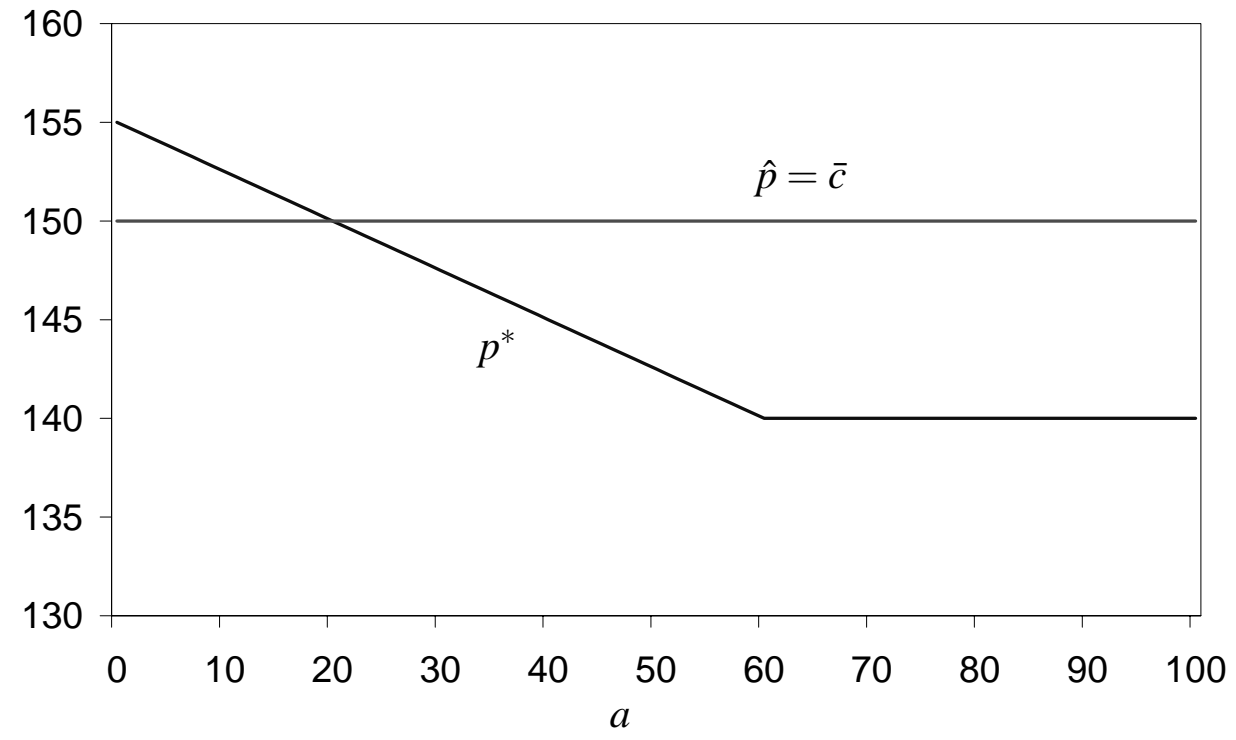

Figure 1: The equilibrium price under the integration approach, $\hat{a}<\tilde{a}$

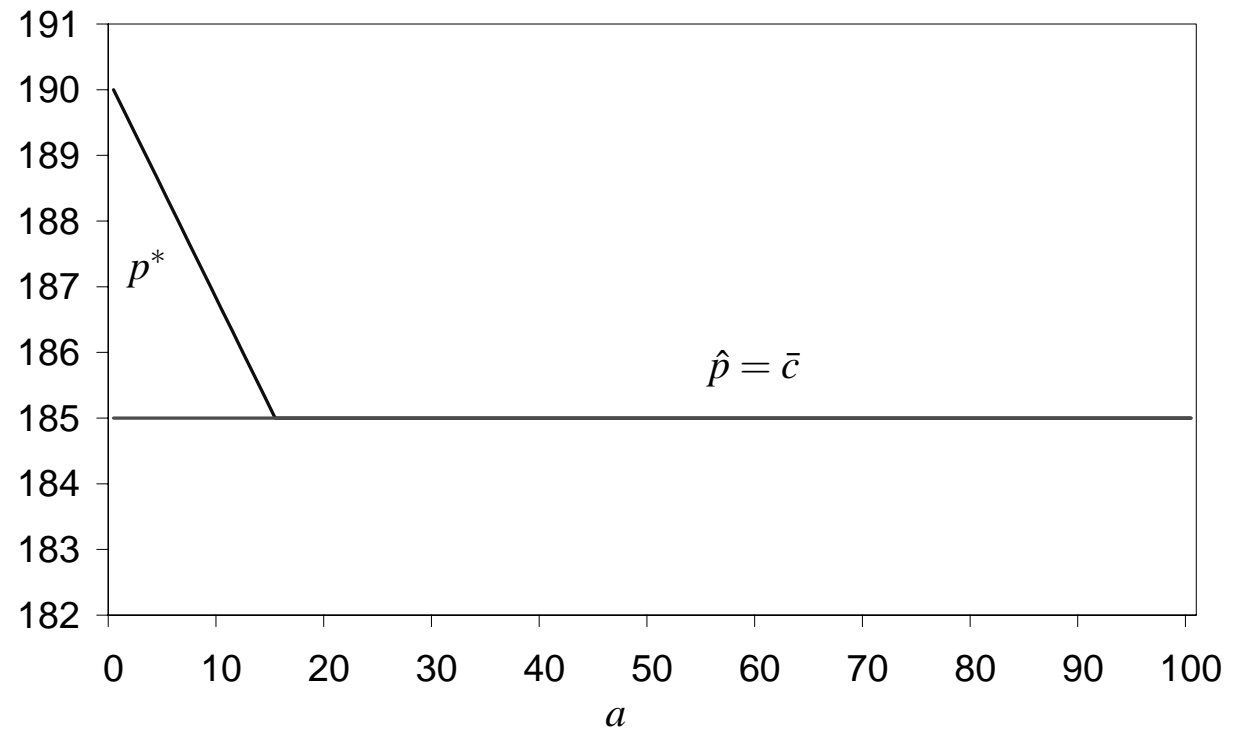

Figure 2: The equilibrium price under the integration approach, $\hat{a}>\tilde{a}$ 
For $a<\tilde{a}$, we obtain case (2). As long as $a<\hat{a}$ we have $p^{*}>\bar{c}$. For $\hat{a}<a<\tilde{a}$, however, the mark-up is so high that the equilibrium falls below $\bar{c}$. At $a=\tilde{a}$ the equilibrium price curve has a kink and case (4) arises for $a \geq \tilde{a}$. The maximum mark-up cannot be enforced because it is profitable for other insurers to make $H 1$ types a fair offer with the total price $p_{4}+P_{4}^{H}=c^{H}+C^{H}$. Thus, increases in $a$ do not lead to a higher mark-up and therefore do not affect the equilibrium price $p^{*}$.

The requirements of Proposition 3 may not always be fulfilled. This is shown in Figure 2 which relies on the same parameters as Figure 1 except for $c^{L}=170$ which implies $\bar{c}=185, \tilde{a}=15$ and $\hat{a}=20$. Thus, $\hat{a}<a<\tilde{a}$ is not possible because $\hat{a}>\tilde{a}$. Cases (1) and (3) apply. Since cross-subsidies from low risk types are much smaller than in the first example, the restriction $P^{L} \geq m$ is not binding and $L 1$-types pay a total fair price $p^{*}+P^{L}=c^{L}+C^{L}$.

For $a<\tilde{a}$, we find that case (1) with $p^{*}>\bar{c}$ prevails. At $a \geq \tilde{a}$, the maximum mark-up cannot be enforced anymore. Case (3) arises in which $H 1$-types receive a fair offer with the total price $p_{3}+P_{3}^{H}=c^{H}+C^{H}$. Since $L 1$-types do not pay cross-subsidies anyhow, both $L 1$ - and $H 1$-types pay a fair price. The remaining cross-subsides between $L 0$ - and $H 0$-types lead to an equilibrium price $p^{*}=\bar{c}$ for $a \geq \tilde{a}$.

Our results allow us to determine who is better off and who is worse off under the integration approach compared to the separation approach:

\section{- H0-types}

$H 0$-types only buy the basic benefit under both regimes. Therefore, they are worse off if $p^{*}<\bar{c}$, i.e. in case (1) and in case (2) if $a<\hat{a}$. They are better off in case (4) and in case (2) if $a>\hat{a}$. Finally, they are equally well off in case (3) and in case (2) if $a=\hat{a}$.

\section{- L0-types}

For $L 0$-types the same reasoning applies as for $H 0$-types. Thus, they are worse off in case (1) and in case (2) if $a<\hat{a}$. They are better off in case (4) and in case (2) if $a>\hat{a}$. In case (3) and in case (2) if $a=\hat{a}$, they are as well off as under the separation approach.

\section{- H1-types}

If $H 1$-types pay the maximum mark-up, they are worse off if $p^{*}>\bar{c}$. Thus, $H 1$-types are always worse off in case (1). In case (2) it depends on whether 


\begin{tabular}{lcccc} 
Case & L0-types & H0-types & L1-types & H1-types \\
\hline (1) & - & - & + & - \\
$(2) a<\hat{a}$ & - & - & + & - \\
$\quad a=\hat{a}$ & 0 & 0 & + & 0 \\
$\quad a>\hat{a}$ & + & + & + & + \\
$(3)$ & 0 & 0 & + & + \\
$(4)$ & + & + & + & +
\end{tabular}

Table 2: Utilities in the integration compared to the separation approach

$p_{2}$ is larger or smaller than $\bar{c}$, i.e. on $a \lessgtr \hat{a}$. In cases (3) and (4), the maximum mark-up cannot be enforced, i.e. $P^{H}<C^{H}+a$. Since in these cases we have $p^{*} \leq \bar{c}, H 1$-types must therefore be better off.

\section{- L1-types}

L1-types are always better off if $m<C^{L}+a$ : In cases (1) and (3) they pay a total price of $p+P^{L}=c^{L}+C^{L}$ since the lower bound for $P^{L}$ is not binding. This is smaller than $\bar{c}+C^{L}+a$, the total price they pay under the separating approach. In case (2), they pay $p_{2}+m=\bar{c}+\mu \lambda C^{L}+(1-\mu \lambda) m-\mu(1-\lambda) a$ which is also smaller than $\bar{c}+C^{L}+a$ if $m<C^{L}+a$. In case (4), the total price equals $p_{4}+m$. Since $p_{4}<\bar{c}, L 1$-types must be better off if $m<C^{L}+a$.

Table 2 summarizes the utilities under the integration approach compared to the separation approach if $m<C^{L}+a$. We conclude:

- in cases (1) and (2), the two regimes may not be comparable with respect to the Pareto-criterion. In case (1), L1-types are better off at the expense of all other individuals. This can be explained as follows: insurers use the complementary benefit to attract $L 1$-types. Since case (1) can only arise if $a$ is low, there are little or, for $a=0$, no gains in terms of saved administrative costs. Thus, all others are worse off because $L 1$-types pay less cross-subsidies to finance the basic benefit for both $H$-types. This effect can also dominate in case (2) if $a$ is lower than $\hat{a}$. For larger $a$, however, the integration approach is Pareto-superior.

- in cases (3) and (4), the integration approach is Pareto-superior. In case (3), all individuals with a preference for the complementary benefit are better off, in case (4) even everyone benefits from the integration of the benefits. 
Interestingly, this result holds in spite of lower cross-subsidies by L1-types. It can be explained by the savings on administrative cost for $H 1$-types and by the fact that $H 1$-types always pay a mark-up for the complementary benefit. The anticipated profits for insurer at stage 2 lead to a lower equilibrium price for the basic benefit at stage 1 . Furthermore, L1-types continue to pay cross-subsidies in case (4).

\section{The minimum price for the complementary benefit}

The minimum price $m$ determines the maximum discount which insurers can give to L1-types. As long as such a discount is possible, the integration approach may lead to a higher price for the basic benefit than the separation approach:

Proposition 4: If a discount to low risk types is possible $\left(m<C^{L}\right)$ and administrative costs a are smaller than $\min \{\tilde{a} ; \hat{a}\}$, then the equilibrium price is larger under the integration approach.

Proof: By (8), we have $\hat{a} \equiv \lambda(1-\lambda)^{-1}\left(C^{L}-m\right)$. Thus $a<\hat{a}$ is only possible if $m<C^{L}$. Furthermore if $a<\tilde{a}$, then only cases (1) or (2) can arise. In case (1), we always have $p^{*}>\bar{c}$. In case (2), we have $p^{*}>\bar{c} \Leftrightarrow a<\hat{a}$ by condition (8).

The economic intuition of this result is that the discount reduces the cross-subsidies to $H 0$-, LO- and H1-types. As long as $a$ and therefore the mark-up for $H 1$-types is not sufficiently high to induce a countervailing reduction of $p^{*}$, this price is higher than under the separation approach.

However, if the regulator sets $m$ equal to $C^{L}$ and therefore rules out a discount for L1-types, risk-selection is not possible with respect to the complementary benefit. Instead, everyone profits from saved administrative costs:

Proposition 5: If administrative costs are positive and the minimum price for the complementary benefit rules out a discount to low risk types $\left(m=C^{L}\right)$, then the integration approach leads to a lower equilibrium price for the basic benefit and is Pareto-superior to the separating approach. 
Proof: Assume first

$$
c^{H}-c^{L}>\min \left\{\frac{a}{\lambda},\left[\frac{1-\mu}{\lambda}+\mu\right] a+\mu\left(C^{L}-m\right)\right\} .
$$

By Proposition 2, we have $p^{*}=\min \left\{p_{1}, p_{2}\right\}$. Since $m=C^{L}$ implies

$$
p_{2}=\bar{c}-\mu(1-\lambda) a<\bar{c},
$$

we must have $p^{*}<\bar{c}$ if $a>0$.

Now suppose

$$
c^{H}-c^{L} \leq \min \left\{\frac{a}{\lambda},\left[\frac{1-\mu}{\lambda}+\mu\right] a+\mu\left(C^{L}-m\right)\right\} .
$$

which is only possible if $a>\tilde{a}>0$ (see conditions (5) and (6)). By Proposition 2, we have $p^{*}=\min \left\{p_{3}, p_{4}\right\} . m=C^{L}$ implies

$$
p_{4}=\bar{c}-\frac{\mu \lambda}{1-\mu(1-\lambda)}(1-\lambda)\left(c^{H}-c^{L}\right)<\bar{c} .
$$

Therefore $p^{*}<\bar{c}$.

Figure 3 shows the equilibrium price for $m=C^{L}$. All parameters are as in Figure 1 except for $m$. Now the equilibrium price is always below $\bar{c}$ for $a>0$ and everyone is better off under the integration approach. Note that in this model $p^{*}$ could furthermore be decreased if $m$ is raised as long as L1-types continue to buy the complementary benefit. However, this result would not hold in a model with more preference types. Then a minimum price above the cost of the complementary benefit for low risk-types leads to a trade-off because some individuals will not buy complementary insurance even though it would be efficient for them to do so.

Proposition 5 should also be interpreted with caution because it requires that a minimum price is enforced, i.e. it has to be controlled that individuals actually pay $m$. Although risk-selection is always possible by lowering the price for low-risks, this problem is particulary acute under the integration approach because insurers are allowed to conduct direct price negotiations with their insured. Nevertheless, Proposition 5 demonstrates that in principle minimum price regulation allows to spread the benefits from the integration approach more evenly among the whole population.

A further way to regulate the health insurance market is to prohibit that losses from selling complementary benefits are financed by profits from basic benefits. 


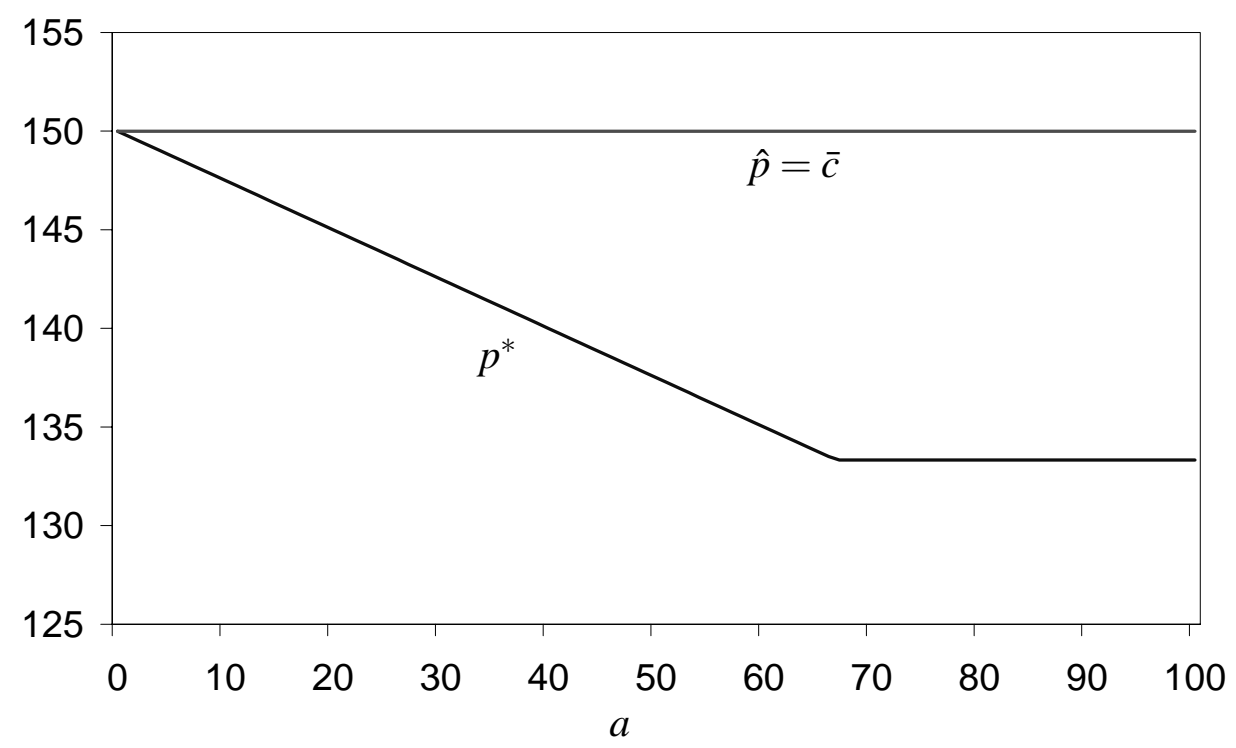

Figure 3: The equilibrium price for $m=C^{L}$

If this regulation is enforced, then the price for the basic benefit could not exceed average costs of the basic benefit and $H 0, H 1$ - and $L 0$-types could never be worse off compared to the separation approach. However, this approach faces a similar problem as enforcing a minimum price because it also requires extensive monitoring of insurers.

\section{Conclusion}

An important public concern with respect to health care is to ensure that everyone has access to a basic benefit package. Furthermore, those who would like to top up their coverage should be able to purchase complementary insurance. This paper has examined two different approaches to reach these objectives. Both require insurers to charge a uniform premium for the basic benefit. Risk-based premiums are allowed for the complementary benefit. Under the separation approach, the benefits must be offered by different insurers. The integration approach practiced in Switzerland, however, permits insurers to sell both benefits. This creates incentives for insurers to risk-select by selling complementary insurance to low risks at a discount. On the other hand, high risks must pay a mark-up when buying the 
complementary benefit from their insurer. Competition between insurers translates this mark-up in a lower price for the basic benefit. Thus, all individuals may potentially benefit from lower administrative costs under the integration approach.

For both approaches, we determined the equilibrium prices. We showed that the cost savings under the integration approach can overcompensate the loss of crosssubsidies from low-risk types due to risk-selection and lead to a lower price of the basic benefit. In this case, everybody is better off under the integration approach. For low levels of cost savings, however, the integration approach is not Paretocomparable to the separation approach. Low-risk types with a preference for the complementary benefit are better off at the expense of all other individuals. We also demonstrated that a minimum price for the complementary benefit can in principle be useful in spreading the cost savings of the integration approach among all insured. However, it remains unclear how effective this regulation can be in practice.

Further research might be dedicated to an empirical analysis of the Swiss health insurance system. So far, empirical studies of the Swiss health insurance system have dealt mainly with the effects of voluntary coinsurance. Schellhorn (2001) and Werblow and Felder (2003) find that low-risk types tend to choose contracts with higher deductibles which shows that risk-selection is a potential problem in the Swiss health insurance market. A first step to see whether this result also applies to the market for complementary insurance could be a study of the relationship between risk and insurance premiums. For the market for general health insurance in the United States, such a study has been performed by Pauly and Herring (1999). Although premiums tend to vary with the risk type, they find no rigid link between risk and premiums. ${ }^{9}$ It would be interesting to find out whether this result also holds for Switzerland. In particular, an important question is whether insurers sell the complementary benefit at a discount to low risks and with a markup to high risks as the model predicts. Furthermore, estimates of the cost savings under the integration approach would be useful in assessing the pros and cons of the two approaches. ${ }^{10}$

\footnotetext{
${ }^{9}$ See Pauly and Herring (1999, p. 52-53).

${ }^{10}$ The precise content of complementary coverage may matter for the potential cost savings under the integration approach. In particular, it can make a difference whether complementary coverage includes hotel amenities or coverage of additional treatments depending on where economies of scope are stronger.
} 


\section{Appendix}

\section{Proof of Proposition 2}

\section{The price offer at stage 2}

To determine the price $P^{i}$ for a given price $p$, we note that if it is profitable for one insurer to offer the basic benefit at price $p$ at stage 1 , then it will also be profitable for others. Thus, at stage 2 perfect competition for customers with respect to the complementary benefit will prevail. This implies that the complementary benefit will be priced such that an individual receives a total fair offer $p+P^{i}=c^{i}+C^{i}$ for both benefits of cost-type $i$ unless

(i) the minimum price restriction $P^{i} \geq m$ is violated. This is the case if $P^{i}=c^{i}+C^{i}-p<m$ which is equivalent to $p>c^{i}+C^{i}-m$, or

(ii) a fair offer implies $P^{i}>C^{i}+a$. This is the case if $P^{i}=c^{i}+C^{i}-p>C^{i}+a$ which is equivalent to $p<c^{i}-a$. An individual would not accept this offer because he can obtain the complementary benefit at price $P^{i}=C^{i}+a$ from another insurer. Since the individual has the right to join the insurer in any case, it is therefore optimal for the basic insurer to make an offer $P^{i}=C^{i}+a$ and charge the mark-up $a$. We assume that in this case individuals buy the complementary benefit from their basic insurer.

The price offer for the complementary insurance is therefore given by

$$
P^{i}(p)=\left\{\begin{array}{ccc}
C^{i}+a & \text { if } & p<c^{i}-a \\
c^{i}+C^{i}-p & \text { if } & c^{i}-a \leq p \leq c^{i}+C^{i}-m \\
m & \text { if } & p>c^{i}+C^{i}-m
\end{array} .\right.
$$

Since the maximum price for the complementary benefit is $P^{i}=C^{i}+a$, the fraction $\mu$ of the population with $V=V^{1}$ will buy complementary insurance from a basic insurer.

Equation (A.1) shows the incentives for risk selection at stage 2: L1-types are likely to receive favorable offers since the price for the complementary benefit tends to be lower, the lower $c^{i}$ and $C^{i}$. The reverse holds for $H 1$-types who are not attractive for insurers. 


\section{The price offer at stage 1}

To derive the equilibrium price $p^{*}$, we make the following observations:

1. H0- and $L 0$-types, who do not care about the complementary benefit, will always choose the insurer with the lowest price $p$.

2. Following equation (A.1), the total price $p+P^{i}$ which $L 1$ - and $H 1$-types pay for health insurance depends in the following way on $p$ :

$$
p+P^{i}(p)=\left\{\begin{array}{ccc}
p+C^{i}+a & \text { if } & p<c^{i}-a \\
c^{i}+C^{i} & \text { if } & c^{i}-a \leq p \leq c^{i}+C^{i}-m \\
p+m & \text { if } & p>c^{i}+C^{i}-m
\end{array}\right.
$$

Thus $L 1$ - and $H 1$-types also have an incentive to choose the insurer with the lowest price $p$ unless $c^{i}-a \leq p \leq c^{i}+C^{i}-m$. In this case, they are indifferent. Then, however, equation (A.1) implies that they pay $c^{i}+C^{i}$. They are therefore irrelevant for the profits of an insurer.

According to these observations, the equilibrium price $p^{*}$ is the lowest price which leads to nonnegative expected profits while providing the basic benefit for everyone and the complementary benefit for those with $V=V^{1}$. The corresponding nonnegative expected profit condition is

$$
p+\mu \lambda P^{L}+\mu(1-\lambda) P^{H} \geq \bar{c}+\mu \lambda C^{L}+\mu(1-\lambda) C^{H} .
$$

The second stage prices are given by equation (A.1). To determine the equilibrium, we need to consider for each type whether the lower bound $\left(P^{i}=m\right)$ and the upper bound $\left(P^{i}=C^{i}+a\right)$ for the price of the complementary benefit (see equation (A.1)) are binding. This is determined by the conditions

$$
p>c^{i}+C^{i}-m \text { and } p<c^{i}-a, \quad i=L, H .
$$

It is not difficult to show that in an equilibrium neither the upper bound for $L 1$ types (i.e. we must have $p \geq c^{L}-a$ ) nor the lower bound for $H 1$-types (i.e. we must have $p \leq c^{H}+C^{H}-m$ ) is binding if $m<C^{H}$. We therefore concentrate on the four cases given in Table 3 .

For each case, we determine under what conditions a price $p$ for the basic benefit exists which leads to nonnegative expected profits. 


\begin{tabular}{lccll} 
Case & $\begin{array}{c}\text { Upper } \\
\text { bound } H 1 \\
\text { binding }\end{array}$ & $\begin{array}{c}\text { Lower } \\
\text { bound } L 1 \\
\text { binding }\end{array}$ & \multicolumn{1}{c}{$P^{H}$} & \multicolumn{1}{c}{$P^{L}$} \\
\hline (1) & $\mathrm{y}$ & $\mathrm{n}$ & $C^{H}+a$ & $c^{L}+C^{L}-p$ \\
$(2)$ & $\mathrm{y}$ & $\mathrm{y}$ & $C^{H}+a$ & $m$ \\
$(3)$ & $\mathrm{n}$ & $\mathrm{n}$ & $c^{H}+C^{H}-p$ & $c^{L}+C^{L}-p$ \\
$(4)$ & $\mathrm{n}$ & $\mathrm{y}$ & $c^{H}+C^{H}-p$ & $m$
\end{tabular}

Table 3: Possible cases in equilibrium

\section{Case (1):}

Inserting for $P^{H}$ and $P^{L}$ from Table 3 into the nonnegative profit constraint (A.3) and simplifying yields

$$
p \geq \bar{c}+\frac{\mu(1-\lambda)}{1-\mu \lambda}\left[\lambda\left(c^{H}-c^{L}\right)-a\right] \equiv p_{1} .
$$

Since the upper bound for $H 1$-types is binding, we have

$$
p_{1}<c^{H}-a \quad \Leftrightarrow \quad c^{H}-c^{L}>\frac{a}{\lambda} \equiv r_{1} .
$$

The lower bound for L1-types is not binding, which implies

$$
p_{1} \leq c^{L}+C^{L}-m \quad \Leftrightarrow \quad c^{H}-c^{L} \leq \frac{1-\mu \lambda}{1-\lambda}\left(C^{L}-m\right)+\mu a \equiv r_{2} .
$$

Case (2):

Inserting for $P^{H}$ and $P^{L}$ from Table 3 into (A.3) and simplifying yields

$$
p \geq \bar{c}+\mu\left[\lambda\left(C^{L}-m\right)-(1-\lambda) a\right] \equiv p_{2} .
$$

The upper bound for $H 1$-types is binding, which requires

$$
p_{2}<c^{H}-a \Leftrightarrow c^{H}-c^{L}>\left[\frac{1-\mu}{\lambda}+\mu\right] a+\mu\left(C^{L}-m\right) \equiv r_{3} .
$$

The lower bound for L1-types is also binding in case (2). Hence,

$$
p_{2}>c^{L}+C^{L}-m \quad \Leftrightarrow \quad c^{H}-c^{L}>\frac{1-\mu \lambda}{1-\lambda}\left(C^{L}-m\right)+\mu a=r_{2} .
$$




\section{Case (3):}

Inserting for $P^{H}$ and $P^{L}$ in (A.3) and simplifying yields

$$
p \geq \bar{c} \equiv p_{3}
$$

Since the upper bound for $H 1$-types is not binding, we have

$$
p_{3} \geq c^{H}-a \quad \Leftrightarrow \quad c^{H}-c^{L} \leq \frac{a}{\lambda}=r_{1}
$$

The lower bound for L1-types is not binding, which yields

$$
p_{3} \leq c^{L}+C^{L}-m \quad \Leftrightarrow \quad c^{H}-c^{L} \leq \frac{C^{L}-m}{1-\lambda} \equiv r_{4} .
$$

\section{Case (4):}

Inserting for $P^{H}$ and $P^{L}$ in (A.3) and simplifying yields

$$
p \geq \bar{c}+\frac{\mu \lambda}{1-\mu(1-\lambda)}\left[C^{L}-m-(1-\lambda)\left(c^{H}-c^{L}\right)\right] \equiv p_{4} .
$$

The upper bound for H1-types is not binding. Hence,

$$
p_{4} \geq c^{H}-a \Leftrightarrow c^{H}-c^{L} \leq\left[\frac{1-\mu}{\lambda}+\mu\right] a+\mu\left(C^{L}-m\right)=r_{3} .
$$

The lower bound for L1-types is also not binding, which implies

$$
p_{4}>c^{L}+C^{L}-m \quad \Leftrightarrow \quad c^{H}-c^{L}>\frac{C^{L}-m}{1-\lambda}=r_{4} .
$$

\section{Deriving the equilibrium prices}

Based on conditions (A.5) to (A.15), we can derive the equilibrium price of the basic benefit, i.e. the lowest price yielding zero expected profits which is compatible with these conditions. To do so, we make the distinction

$$
r_{1}=\frac{a}{\lambda} \gtreqless\left[\frac{1-\mu}{\lambda}+\mu\right] a+\mu\left(C^{L}-m\right)=r_{3} .
$$


First, we assume $r_{1}<r_{3}$. Using conditions (A.5) to (A.15), we obtain the unambiguous results

$$
p^{*}=\left\{\begin{array}{lll}
p_{1} & \text { if } & c^{H}-c^{L}>r_{3} \text { and } c^{H}-c^{L} \leq r_{2} \\
p_{2} & \text { if } & c^{H}-c^{L}>r_{3} \text { and } c^{H}-c^{L}>r_{2} \\
p_{3} & \text { if } & c^{H}-c^{L} \leq r_{1} \text { and } c^{H}-c^{L} \leq r_{4} \\
p_{4} & \text { if } & c^{H}-c^{L} \leq r_{1} \text { and } c^{H}-c^{L}>r_{4}
\end{array} .\right.
$$

If $r_{1}<c^{H}-c^{L} \leq r_{3}$, then cases (2) and (3) can be excluded whereas cases (1) and (4) can in principle both apply. However, by (A.5) and (A.14) we have $p_{1}<$ $c^{H}-a \leq p_{4}$. Since the lower price prevails in an equilibrium, this implies $p^{*}=p_{1}$.

Second, we assume $r_{1} \geq r_{3}$. Using conditions (A.5) to (A.15), we obtain the unambiguous results

$$
p^{*}=\left\{\begin{array}{lll}
p_{1} & \text { if } & c^{H}-c^{L}>r_{1} \text { and } c^{H}-c^{L} \leq r_{2} \\
p_{2} & \text { if } & c^{H}-c^{L}>r_{1} \text { and } c^{H}-c^{L}>r_{2} \\
p_{3} & \text { if } & c^{H}-c^{L} \leq r_{3} \text { and } c^{H}-c^{L} \leq r_{4} \\
p_{4} & \text { if } & c^{H}-c^{L} \leq r_{3} \text { and } c^{H}-c^{L}>r_{4}
\end{array} .\right.
$$

If $r_{3}<c^{H}-c^{L} \leq r_{1}$, then cases (1) and (4) can be excluded whereas cases (2) and (3) can in principle both apply. However, by (A.8) and (A.11) we have $p_{2}<$ $c^{H}-a \leq p_{3}$, which implies $p^{*}=p_{2}$.

Noting that $c^{H}-c^{L}>r_{2} \Leftrightarrow p_{1}>p_{2}, r_{3}<c^{H}-c^{L} \leq r_{1} \Leftrightarrow p_{1}>p_{2}$ (this follows from conditions (A.5) and (A.8) which imply $p_{1}>c^{H}-a \geq p_{2}$ ) and $c^{H}-c^{L}>$ $r_{4} \Leftrightarrow p_{3}>p_{4}$, the equilibrium price can finally be expressed as

$$
p^{*}=\left\{\begin{array}{lll}
\min \left\{p_{1}, p_{2}\right\} & \text { if } & c^{H}-c^{L}>\min \left\{r_{1}, r_{3}\right\} \\
\min \left\{p_{3}, p_{4}\right\} & \text { if } & c^{H}-c^{L} \leq \min \left\{r_{1}, r_{3}\right\}
\end{array} .\right.
$$

Together with the definition of cases (1) to (4) in Table 3, prices $p_{z}, z=1,2,3,4$, (see (A.4), (A.7), (A.10) and (A.13)) and $r_{1}$ and $r_{3}$ (see (A.5) and (A.8)), this establishes Proposition 2. 


\section{References}

Beck, K. (1998). "Kann der Risikoausgleich unterlaufen werden? Analyse der schweizerischen Ausgleichsformel," in Gesellschaft für Recht und Politik im Gesundheitswesen (ed.), Fairneß, Effizienz und Qualität in der Gesundheitsversorgung: was kann der Risikostrukturausgleich dazu leisten? pp. 99-146 Springer: Heidelberg.

Bundesamt für Sozialversicherung (2000). "Veränderungen im Bereich der Zusatzversicherung aufgrund des KVG," BSV-Forschungsbericht 04/00, Bundesamt für Sozialversicherung, Bern.

Colombo, F. (2001). "Towards more choice in social protection? Individual choice of insurer in basic mandatory health insurance in Switzerland," OECD Labour Market and Social Policy - Occasional Paper 53, OECD.

Danzon, P. (2002). "Welfare Effects of Supplementary Insurance: a Comment," Journal of Health Economics 21, 923-926.

Glazer, J., and T. McGuire (2000). "Optimal Risk Adjustment in Markets with Adverse Selection: An Application to Managed Care," American Economic Review 90, 1055-1071.

Kifmann, M. (2002). "Community Rating in Health Insurance and Different Benefit Packages," Journal of Health Economics 21, 719-737.

Mossialos, E., S. Thomson, R. Busse, C. Economou, M. Giannoni-Mazzi, J. Hermesse, T. Hockley, M. M. Hofmarcher, H. Maarse, H. Mikkola, M. Oliveira, M. Rodríguez, S. Sandier, C. Skoglund, P. Ulmann, and K. Vrangbæk (2002). "Voluntary health insurance in the European Union," Report prepared for the directorate general for employment and social affairs of the european commission, http://europa.eu.int/comm/employment_social/soc-prot/social/vhi.pdf.

Pauly, M. (1984). "Is Cream-Skimming a Problem for the Competitive Medical Market?," Journal of Health Economics 3, 87-95.

Pauly, M., P. Danzon, P. Feldstein, and J. Hoff (1992). Responsible National Health Insurance. AEI Press: Washington, D.C.

Pauly, M., and B. Herring (1999). Pooling Health Insurance Risks. American Enterprise Institute: Washington, D.C. 
Schellhorn, M. (2001). "The Effect of Variable Health Insurance Deductibles on the Demand for Physician Visits," Health Economics 10, 441-456.

Werblow, A., and S. Felder (2003). "Der Einfluss von freiwilligen Selbstbehalten in der gesetzlichen Krankenversicherung: Evidenz aus der Schweiz," Schmollers Jahrbuch 123, 235-264. 